\title{
Integrated hybrid Raman/fiber Bragg grating interrogation scheme for distributed temperature and point dynamic strain measurements
}

\author{
Farhan Zaidi, ${ }^{1}$ Tiziano Nannipieri, ${ }^{1}$ Marcelo A. Soto, ${ }^{1,2}$ Alessandro Signorini, ${ }^{1, *}$ \\ Gabriele Bolognini, ${ }^{3}$ and Fabrizio Di Pasquale ${ }^{1}$ \\ 'Scuola Superiore Sant'Anna, Institute of Communication, Information and Perception Technologies, \\ via G. Moruzzi 1, Pisa 56124, Italy \\ ${ }^{2}$ Permanent address at the Ecole Polytechnique Fédérale de Lausanne, Institute of Electrical \\ Engineering, SCI STI LT, Station 11, Lausanne 1015, Switzerland \\ ${ }^{3}$ Consiglio Nazionale delle Ricerche, Institute for Microelectronics and Microsystems, \\ via P. Gobetti 101, Bologna 40129, Italy \\ *Corresponding author: al.signorini@sssup.it
}

Received 13 July 2012; revised 6 September 2012; accepted 13 September 2012; posted 18 September 2012 (Doc. ID 172577); published 16 October 2012

\begin{abstract}
We propose and experimentally demonstrate the feasibility of an integrated hybrid optical fiber sensing interrogation technique that efficiently combines distributed Raman-based temperature sensing with fiber Bragg grating (FBG)-based dynamic strain measurements. The proposed sensing system is highly integrated, making use of a common optical source/receiver block and exploiting the advantages of both (distributed and point) sensing technologies simultaneously. A multimode fiber is used for distributed temperature sensing, and a pair of FBGs in each discrete sensing point, partially overlapped in the spectral domain, allows for temperature-independent discrete strain measurements. Experimental results report a dynamic strain resolution of $7.8 \mathrm{n} \varepsilon / \sqrt{ } \mathrm{Hz}$ within a full range of $1700 \mu \varepsilon$ and a distributed temperature resolution of $1^{\circ} \mathrm{C}$ at $20 \mathrm{~km}$ distance with $2.7 \mathrm{~m}$ spatial resolution. () 2012 Optical Society of America

OCIS codes: $\quad 060.2370,190.5650,060.3735,280.4788$.
\end{abstract}

\section{Introduction}

During the past decade, fiber-optic sensors have emerged as a promising industrial technology with respect to conventional electrical sensors due to their unique advantages such as electromagnetic interference immunity, high sensitivity to measurand variations, and environmental ruggedness as well as the possibility of integration within smart structures and materials, allowing their effective use in many industrial applications [1-3]. In principle, optical fiber sensors can be fundamentally classified into two

$1559-128 \mathrm{X} / 12 / 307268-08 \$ 15.00 / 0$

(C) 2012 Optical Society of America categories: distributed and point (or discrete) sensors [4]. Distributed sensing technology is based on scattering effects [5] such as Raman or Brillouin scattering, in which the measurand is continuously monitored along an optical fiber, which acts itself as the sensing element. The second type of sensors involves the use of point fiber sensing elements, such as fiber Bragg gratings (FBGs), in which the measurement takes place at particular spatial location along the fiber [6]. To overcome the limitations imposed by the cost and interrogation complexity of many point sensors, quasi-distributed sensing can be implemented based on array of FBGs employing different multiplexing techniques [7]. Among all multiplexing methods, time division multiplexing 
(TDM) is one of the most promising techniques since it makes efficient use of the optical spectrum, allowing for several FBG sensors to be employed in the same wavelength window and providing at the same time measurements with a good dynamic range [8].

While Raman-based distributed temperature sensors (RDTSs) constitute to date the most successful and widely adopted technology for distributed sensing $[1,9]$, allowing for quasi-static measurements with typical time scales of tens of seconds or few minutes, FBG-based sensing is the most commonly employed technique for point measurements, allowing for multiplexed discrete sensing with dynamic measurement capabilities up to several kilohertz [6]; the range of their application fields spans from transportation to industrial power plants monitoring and oil and gas leakage detection in wells and pipelines.

In many applications simultaneous dynamic and distributed measurement would be highly desired, as for example, in petrochemical industrial plant monitoring where a slow temperature profiling throughout the plant, useful for process control, can be effectively associated to dynamic strain information at specific critical points, useful to early detect possible cracks in pipelines. For such applications, an efficient sensor system should be devised combining distributed (static) temperature measurements and discrete (dynamic) strain sensing, thus providing detection of anomalous operating conditions (i.e., overheating, leakages, and fire along a pipeline or within a plant) and a simultaneous dynamic monitoring of the structure (i.e., vibrations in proximity of critical locations such as joints along a pipeline or a plant). It must be considered that, in most industrial applications, due to significant thermal inertia of the fiber cabling (often involving polymer sheaths, rodent protection, and external armoring in addition to the coated optical fiber itself), local temperature exhibits significant variations only on time scales extending over the second scale (often being as slow as many tens of seconds at least). To this extent, monitoring with Raman distributed temperature sensing with a temporal response of a few seconds would provide a calibration on local temperature that can be effectively used to accurately monitor the dynamic FBG strain measurement.

Both RDTSs and FBG-based sensing provide stable and reliable measurements when exploited in time domain, and therefore, they can be good candidates to be integrated in a hybrid sensor that utilizes all functionalities of both sensing technologies simultaneously.

In this paper we propose a highly integrated hybrid sensing system (patent pending) that effectively combines the advantages of both RDTSs and TDM-FBG-based dynamic sensing by fully exploiting their respective specific functionalities.

An integrated sensing unit based on a shared laser source and receiver block has been developed, allowing for distributed measurements up to $20 \mathrm{~km}$ distance as well as dynamic strain sensing with a Nyquist limit up to $2.5 \mathrm{kHz}$.
The proposed technique provides a multifunctional and cost-effective solution in several industrial sectors such as in transportation, pipelines, and industrial plant monitoring.

\section{Theory: Integrated Sensing Approach}

In conventional RDTS systems, optical pulses are launched into a sensing fiber so that, using optical time-domain reflectometry (OTDR) techniques [10], the spontaneous Raman scattering (SpRS) light that is backscattered along the fiber is measured as a function of the distance. The forward-propagating light pulse generates two SpRS components: the so-called Stokes and anti-Stokes lights. Although only the intensity of the anti-Stokes SpRS component strongly depends on the fiber temperature, a normalization with a temperature-independent OTDR trace, such as the Stokes SpRS or Rayleigh component, must be carried out to distinguish real temperature changes from local fiber loss variations $[10,11]$. In our implemented RDTS system, trace normalization has been carried out by using Stokes light; in such schemes the anti-Stokes over Stokes intensity ratio is employed, providing a temperature sensitivity that can be expressed as

$$
R(z, T)=C \cdot\left(\frac{\lambda_{S}}{\lambda_{\mathrm{AS}}}\right)^{4} \exp \left[-\frac{h \Delta \nu}{k T(z)}\right],
$$

where $T(z)$ is the fiber temperature, $C$ is a constant dependent on fiber differential loss and receiver conditions, $\lambda_{S}$ and $\lambda_{\mathrm{AS}}$ are the Stokes and anti-Stokes wavelengths, respectively, $h$ is the Planck constant, $\Delta \nu$ is the frequency separation, and $k$ is the Boltzmann constant.

The absolute temperature profile $T(z)$ is found through a reference temperature profile $T_{0}(z)$ obtained from an initial calibration procedure involving an off-field calibration of fiber response at known temperature conditions and a subsequent infield calibration requiring external measurement of temperature only at some fixed points (typically two points) to apply suitable corrections on fiber response impacting the ratio that might arise during deployment (mainly impacting slope of reference ratio). The absolute temperature is then found using the following equation:

$$
T(z)^{-1}=T_{0}(z)^{-1}-\frac{k}{h \Delta \nu} \ln \left(\frac{R(z, T)}{R\left(z, T_{0}\right)}\right) .
$$

Equation (2) provides a $T(z)$ profile that is highly robust with respect to variations in optoelectronic parameters of the receiver stage, fiber conditions, and losses as well as laser power fluctuations.

On the other hand, FBGs are point fiber sensors acting as band-reflect optical filters; the reflection bandwidth, centered at the Bragg wavelength $\lambda_{B}$, due to changes in the effective index and grating pitch linked to strain and temperature, exhibits a linear dependence on temperature $(\Delta T)$ and strain variations $(\Delta \varepsilon)[12]$, according to the formula: 


$$
\Delta \lambda_{B}=\lambda_{B}\left[(\alpha+\varsigma) \Delta T+\left(1-p_{\varepsilon}\right) \Delta \varepsilon\right] .
$$

In Eq. (3), $\Delta \lambda_{B}$ is the variation of Bragg wavelength, $\alpha$ and $\varsigma$ are the thermal expansion and thermo-optic coefficients, respectively, and $p_{\varepsilon}$ is the effective photoelastic constant of the fiber material.

The integrated hybrid sensing approach that is proposed in this paper makes use of a single pulsed laser that is simultaneously employed for both distributed Raman sensing and point dynamic FBG interrogation. In particular the system requires the use of two optical fibers (possibly within the same duplex cable): a graded-index multimode fiber (MMF) for Raman-based temperature measurements, and a single-mode fiber (SMF) for FBG-based sensing. Even though a unique SMF could be used for both distributed and discrete sensing, the larger effective area of MMFs allows higher peak power to be launched into the fiber before the onset of nonlinearities, increasing both the Raman backscattered power and the signal-to-noise ratio (SNR) at the receiver. This feature greatly enhances the RDTS sensing capabilities, resulting in a more suitable solution for high-performance, long-range distributed temperature measurements.

In addition, the proposed method employs a timedomain approach for interrogating a pair of FBGs, which are placed close to each other in cascade along the same SMF at each discrete sensing point. The performance of the multiplexed discrete sensor in terms of maximum acceptable cross-talk level, sensor resolution, and measurement range are mainly dictated by FBG reflectivity, FBG bandwidth, and number of discrete sensing points [8]. Regarding this, the value of FBG reflectivity involves a trade-off between sensor point number and performance, since, while low-reflectivity FBGs results in lower backreflected power levels and then lower SNR levels, employing high-reflectivity FBGs (especially when many sensing points are needed) can induce significant penalties arising from multiple FBG reflection effects, thus hindering the sensor performance. In our experiment scheme, thanks to a high-sensitivity receiver simultaneously employed in low-power photodetection for a RDTS, we could ensure adequate SNR levels and sensor performance in terms, e.g., of strain resolution even with low-reflectivity FBGs. Thus, by employing lowreflectivity FBGs and high input power from a rare-earth-doped pulsed laser source, a dense array of many serial discrete sensing points can be placed along the fiber.

The basic principle of the FBG interrogation technique is illustrated in Fig. 1 . The light from a narrowband pulsed laser source (the same source is also used for distributed Raman sensing) is employed to interrogate, at each sensing point, a pair of lowreflectivity Gaussian-apodized FBGs whose reflection spectra have to be symmetrically shifted with respect to the central wavelength of the laser source. Both FBGs at each specific sensing point should be

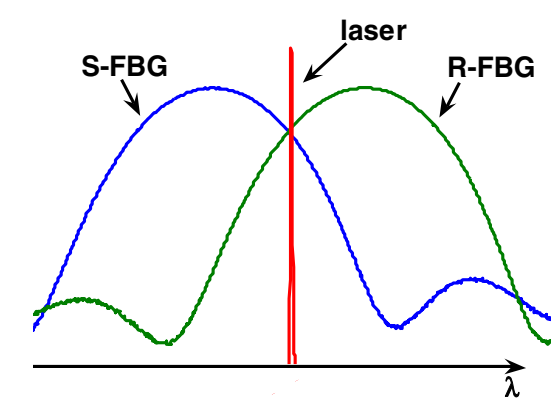

Fig. 1. (Color online) Proposed dynamic strain measurement technique.

closely spaced and spatially separated by a few meter fiber spool within a small form-factor packaged coiling in order for them to be subjected to the same temperature. Only one FBG of the FBG pair should be attached to the structure of interest [the so-called sensing FBG (S-FBG)] and therefore made sensitive to both strain and temperature simultaneously. The other grating, named reference FBG (R-FBG), should be loose and subjected to temperature only, allowing for a strain-independent reference to compensate for optical power variations resulting from changes in local losses or from laser power fluctuations. Since the employed FBG interrogation technique uses the discrete sensors as linear filter to translate wavelength shift into amplitude variations, the maximum effective measurement range for point temperature and strain depends on both the spectral separation between the central wavelengths of the FBG pair and the FBG bandwidth. To take into account the Gaussian shape of the FBG reflectivity spectrum, we have defined a temperature- and strain-dependent interrogation function $\rho\left(\Delta \lambda_{B}\right)$ as the logarithm of the ratio between the integral of the reflected pulses from each FBG,

$$
\rho\left(\Delta \lambda_{B}\right)=K \times \ln \left(\frac{\int_{z_{\mathrm{R}-\mathrm{FBG}}}^{z_{\mathrm{R}-\mathrm{BG}}+\Delta z} I_{\mathrm{RS}-\mathrm{FBG}}\left(\Delta \lambda_{B}, \xi\right) \mathrm{d} \xi}{\int_{z_{\mathrm{S}-\mathrm{FBG}}}^{z_{\mathrm{FBG}}+\Delta z} I_{\mathrm{RS}-\mathrm{FBG}}\left(\Delta \lambda_{B}, \xi\right) \mathrm{d} \xi}\right),
$$

where $I_{\mathrm{RS}-\mathrm{FBG}}\left(\Delta \lambda_{B}, \xi\right)$ is the intensity of the backreflected pulses from FBG pair and depends upon the Bragg wavelength shift $\Delta \lambda_{B}, z_{\mathrm{R}-\mathrm{FBG}}$ and $z_{\mathrm{S}-\mathrm{FBG}}$ are the longitudinal positions of FBGs along the fiber, $\Delta z$ is the spatial extent of the FBG response for given pulse conditions in the RDTS pump, and $K$ is a correction factor that allows for sensor calibration and takes into account any static variation of $\Delta \lambda_{B}$ affecting both R-FBG and S-FBG simultaneously.

It is important to notice that, in this configuration, the impact of strain on the FBG response is different than the impact of temperature. Actually, small or moderate changes of temperature are expected to shift linearly the Gaussian-shaped spectrum of both FBGs as from Eq. (3); hence, the logarithm of interrogation function in Eq. (4) is expected to exhibit a linear behavior with temperature. This linear behavior should only occur within a particular range of temperature, which depends on the spectral 
separation between the central wavelengths of each FBG. On the other hand, if strain is applied, only the spectrum of S-FBG is expected to be shifted; in any case, the value given by Eq. (4) also depends on the spectral position of both FBGs, which is determined by the local temperature affecting both FBGs. This issue leads to a linear strain dependence of the interrogation function with a slightly different slope for every temperature, as reported by the dashed lines in Fig. 2 , showing the slope dependence of interrogation function versus applied strain for different temperature values. To compensate for such effect and obtain a reliable point strain measurement at all operating temperatures, a calibration process has been carried out; in such a process, the temperaturedependent factor $K$ (with $K=1$ at room temperature, $25^{\circ} \mathrm{C}$ in our case) in Eq. (4) is characterized, using the information obtained from the RDTS, to compensate the impact of temperature on the interrogation function. We can observe in Fig. 2 (solid lines) that the temperature-dependent slope of the interrogation function can be easily corrected with a proper characterization of the factor $K$, leading to approximately linear traces with similar slopes, and only affected by an offset at different temperature. It is worth noticing that, since Raman-based distributed temperature measurements are carried out in a (graded-index) MMF, the spatial resolution of the temperature profile is affected by the pulse broadening during light propagating resulting from modal dispersion (increasing along the fiber from the initial value determined by the pulse duration, from $1 \mathrm{~m}$ at fiber input to about $1.4 \mathrm{~m}$ after $20 \mathrm{~km}$ fiber propagation considering the employed standard graded-index fiber). On the other hand, point dynamic strain measurements employ an SMF, and therefore the impact of chromatic dispersion on the pulses and the respective reflected light (from the FBGs) can be considered to be negligible.

\section{Experimental Setup}

The experimental setup is shown schematically in Fig. 3. To implement the low-cost integrated hybrid

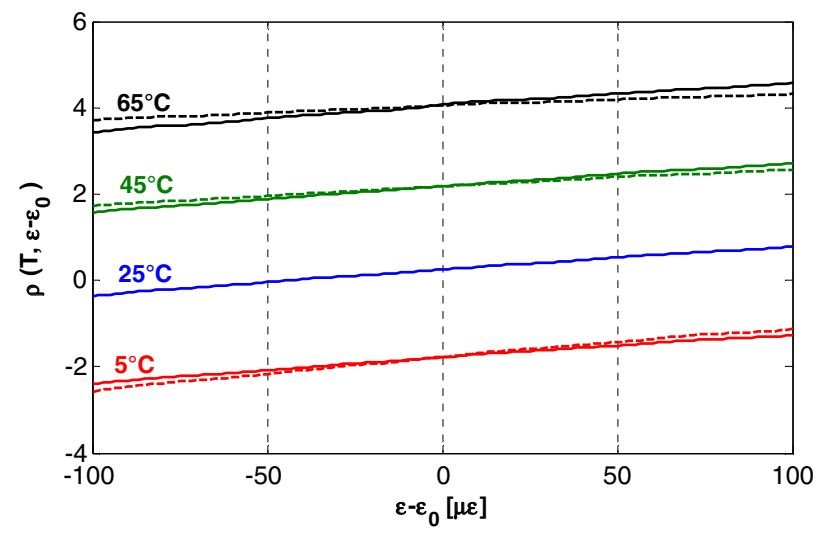

Fig. 2. (Color online) Characterization of interrogation function $\rho$ versus applied strain $\varepsilon-\varepsilon_{0}$ at different temperatures (dashed line, before slope correction; solid line, after slope correction). sensor proposed in this paper, a shared sensor reading/interrogation unit (within the dashed block in Fig. 3) has been developed. The sensor interrogation unit exploits a commercial single rare-earthdoped fiber pulsed laser, centered at $1550.5 \mathrm{~nm}$, and provides $10 \mathrm{~ns}$ pulses with a maximum peak power of $\sim 50 \mathrm{~W}$ at a repetition rate of $5 \mathrm{kHz}$. A couple of variable optical attenuators (VOAs) are placed before the fibers to reduce the peak power input level, thus avoiding the onset of fiber nonlinearities. In addition, an optical splitter is used to couple the pulsed light from the sensing unit into both sensing optical fibers. The routing and filtering stage is composed of an optical circulator along the SMF branch (employed for coupling light into the time-multiplexed FBG pairs and for routing the respective backreflected light onto the receiver stage) and a four-port optical filter (coupling the pulsed pump light into the MMF used in distributed sensing and extracting the backscattered Stokes and anti-Stokes components before the receiver stage).

The integrated receiver block consists of an amplified photodiode array, which is composed of a couple of avalanche photodiodes for Stokes and anti-Stokes light detection and a PIN diode for FBGbackreflected light detection. In addition, a multichannel analog-to-digital converter (ADC) enables simultaneous acquisition of the analog waveforms with $1 \mathrm{GS} / \mathrm{s}$ sampling frequency and subsequent processing by a PC-controlled field programmable gate array-based board. While the laser pulse width and the receiver bandwidth up to $125 \mathrm{MHz}$ in principle allow for a spatial resolution of $1 \mathrm{~m}$, the maximum FBG interrogation rate is limited by the laser repetition rate $(5 \mathrm{kHz}$ imposed by the fiber length) leading to a maximum FBG dynamic strain reconstruction down to $2.5 \mathrm{kHz}$ (Nyquist limit).

The output light from the pulsed laser is coupled into two $20 \mathrm{~km}$ fibers (within the same duplex cable), i.e., an SMF for dynamic strain sensing, and an MMF for distributed temperature sensing. Along the MMF branch, the laser light (single-mode pigtail) is coupled into the filtering block and then into the MMF by an SMF-MMF fiber-mode coupler to reduce higher-order mode leakage. Along the SMF branch for point sensing, we employed an FBG pair with the two FBGs centered at $1549.35 \mathrm{~nm}$ (S-FBG) and $1551.4 \mathrm{~nm}$ (R-FBG), and showing the same nominal peak reflectivity $(5 \%)$ and bandwidth $(2.5 \mathrm{~nm})$. This allows for an expected full-scale temperature measurement range of $170^{\circ} \mathrm{C}$ (when no strain is applied) and a strain measurement range of $\sim 1700 \mu \varepsilon$ (at room temperature) [13]. In case of simultaneous strain and temperature measurements, the total measurement range for both temperature and strain is reduced so that for instance, to realize the strain sensing up to $800 \mu \varepsilon$, the temperature range results to be from $-15^{\circ} \mathrm{C}$ to $65^{\circ} \mathrm{C}$. Both gratings have been placed at $15.2 \mathrm{~km}$ distance and have been spatially separated by $2.5 \mathrm{~m}$ coiled SMF (small form factor). 


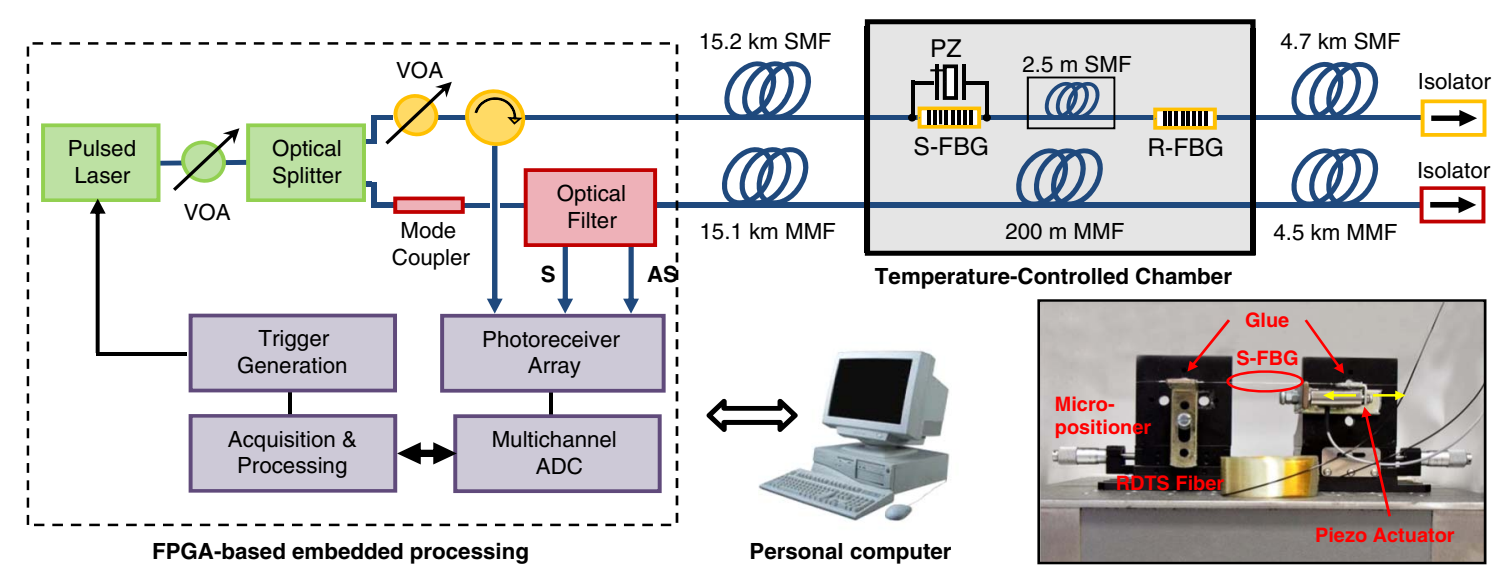

Fig. 3. (Color online) Experimental setup showing the sensor reading unit and the sensing fibers. (Inset) Picture of piezoelectric (PZT) actuation system used to apply dynamic strain to the S-FBG.

\section{Results}

To analyze and validate the capability of our approach to discriminate temperature and strain, we placed $200 \mathrm{~m}$ of MMF and both FBGs inside a temperature-controlled chamber (TCC). Strain was applied only to the S-FBG along the longitudinal direction using a piezoelectric (PZT)-action system (the inset in Fig. 3 shows all the components that have been placed inside the TCC to apply strain together with temperature variations). The S-FBG was actually prestrained to apply dynamic strain effectively and increase the grating compression sensitivity.

To obtain the temperature dependence of the interrogation function in Eq. (4), both gratings have been placed into the TCC at a temperature that was varied from $15^{\circ} \mathrm{C}$ up to $45^{\circ} \mathrm{C}$, shifting the reflectivity spectrum of both S-FBG and R-FBG. Figure 4(a) shows the time-domain traces of the backreflected pulses from both gratings, confirming the
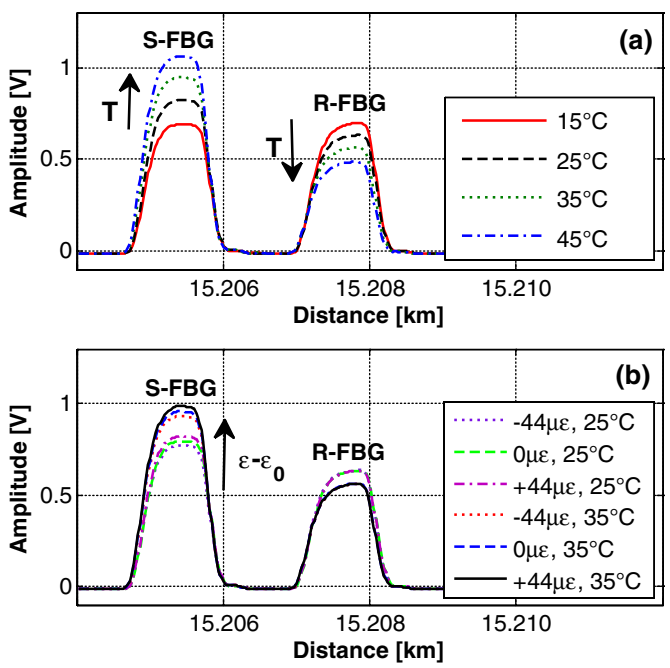

Fig. 4. (Color online) Time-domain traces of FBG-reflected pulses (a) at different temperatures and (b) at different values of applied strain $\varepsilon-\varepsilon_{0}$ and temperature $T$. counteracting effect of temperature on each of them. To further characterize the strain interrogation function, a static strain $(\Delta \varepsilon)$ has also been applied to the S-FBG by varying the PZT from $-44 \mu \varepsilon$ up to $+44 \mu \varepsilon$ at two different temperatures $\left(25^{\circ} \mathrm{C}\right.$ and $\left.35^{\circ} \mathrm{C}\right)$. Figure $4(\mathrm{~b})$ shows the time-domain traces at different strain and temperature values, pointing out the significant strain-temperature cross-sensitivity affecting the measurements.

To compensate for temperature variations and obtain the real dynamic strain, a slope characterization of the interrogation function versus strain was carried out at different temperatures. The experimental results reported in Fig. 5 show the temperature dependence of the interrogation function according to Eq. (4) (with $K=1$ ). The figure clearly shows a linear behavior of the interrogation function against temperature in the presence of a constant applied prestrain $\left(\varepsilon_{0}\right)$ on S-FBG. Simulations have also been carried out using the spectra of the used FBGs and their sensitivity on temperature, resulting in good agreement with the experimental data as shown in Fig. 5. By analyzing Eq. (4), it is possible to find out that, when strain has to be measured, the local temperature introduces an offset in the interrogation function (due to the linear behavior of

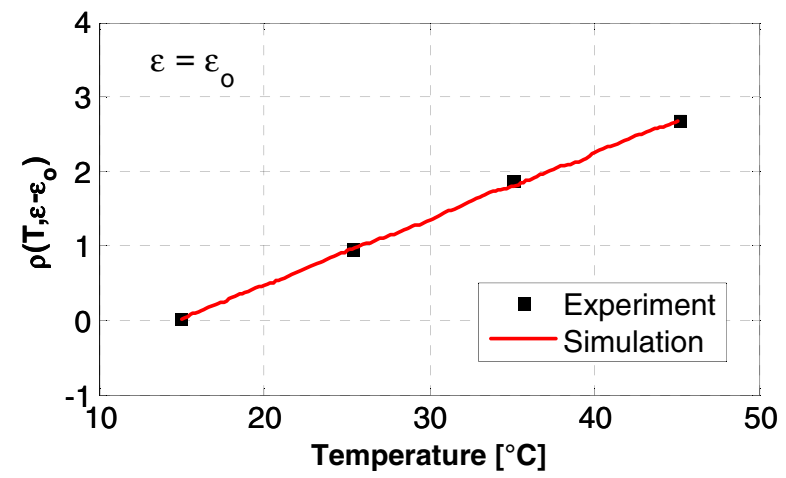

Fig. 5. (Color online) Characterization of the FBG interrogation function $\rho(T, \varepsilon)$ versus temperature with no applied strain $\varepsilon-\varepsilon_{0}$ (squares, experimental data; solid line, simulations). 

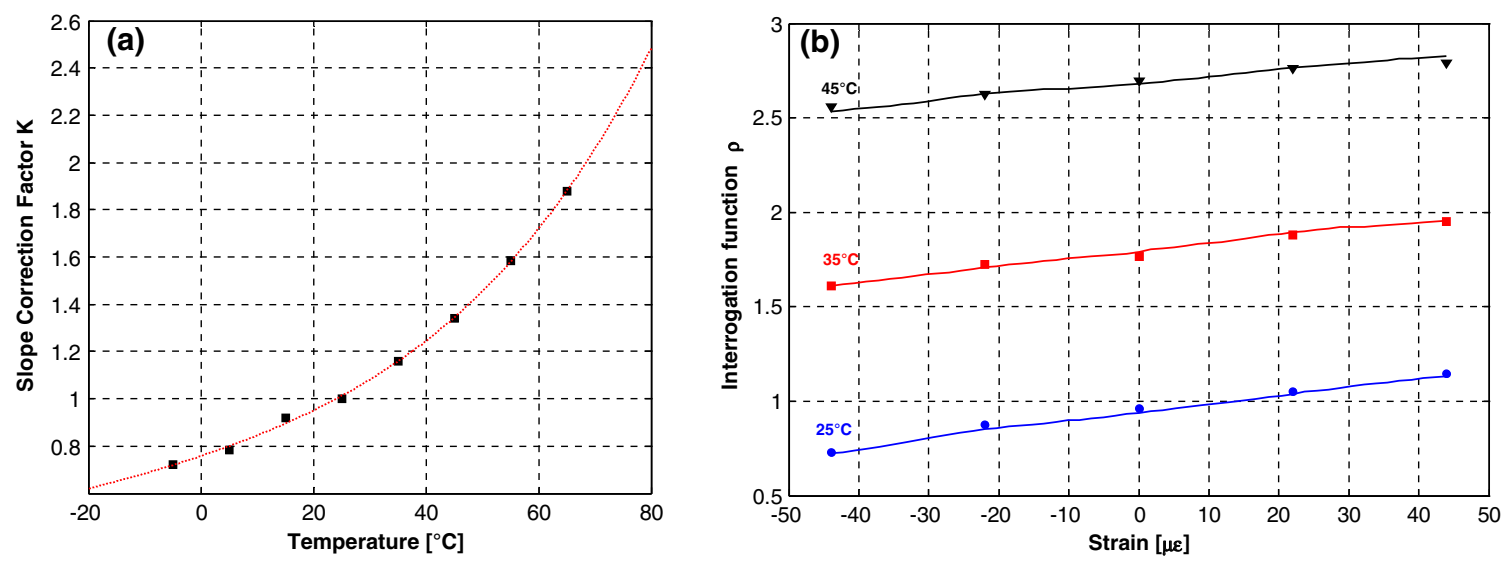

Fig. 6. (Color online) (a) Slope correction factor $K$ for interrogation function versus temperature. (b) Characterization of interrogation function versus applied strain at different temperatures (symbols, experimental data; dotted line, simulations).

Fig. 5) but also changes the slope of the characterization versus strain. As explained in Section 2, to provide a practical and temperature-independent strain estimation algorithm, the temperaturedependent factor $K$ in Eq. (4) has been calibrated so that the strain dependence becomes temperatureinsensitive, with a linear behavior that is characterized by the same slope at different temperatures (as previously explained and shown in Fig. 2). To provide $K$ factor calibration, the correction factor $K$ has been obtained as a function of temperature by increasing the temperature in both gratings and keeping a constant strain; the behavior is reported in Fig. 6(a) (where the local temperature information has been obtained by Raman sensing). We also verified that the correction factor $K$ does not change appreciably if the initial (residual) strain applied to S-FBG varies, thus allowing for a unique calibration for any initial strain value applied to S-FBG. Figure $6(\mathrm{~b})$ shows the corrected interrogation function versus strain at different temperature values, where we can observe a constant slope of the function for different temperatures. Using such a characterization (i.e., a linear behavior with constant slope), the temperatureindependent strain value can be easily estimated

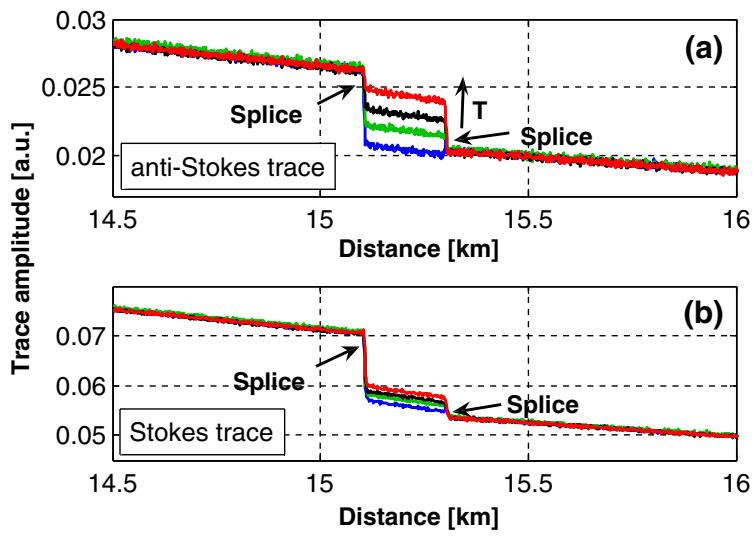

Fig. 7. (Color online) (a) Anti-Stokes and (b) Stokes traces at different TCC temperatures (blue, $15^{\circ} \mathrm{C}$; green, $25^{\circ} \mathrm{C}$; black, $35^{\circ} \mathrm{C}$; red, $45^{\circ} \mathrm{C}$ ). once the local temperature information obtained by Raman sensing is used to compensate for the temperature-induced offsets shown in Fig. 6(b).

To verify the capabilities of the system to perform at the same time distributed temperature sensing and reliable temperature-compensated dynamic strain measurements, the TCC temperature (affecting both gratings) was varied from $15^{\circ} \mathrm{C}$ up to $45^{\circ} \mathrm{C}$ and a sinusoidal dynamic strain $(88 \mu \varepsilon$ peak-to-peak amplitude) was simultaneously applied to S-FBG.

The anti-Stokes and Stokes intensity traces resulting from RDTS (using the above-mentioned measurement setup with $100 \mathrm{k}$ averages and $30 \mathrm{~s}$ total measurement time) are reported in Figs. 7(a) and 7(b), respectively (reporting for clarity the timedomain traces around a $15 \mathrm{~km}$ fiber distance where the TCC is placed).

The RDTS calibration procedure (discussed in Section 2) has been carried out to take into account all possible effects impacting on acquired traces, such as receiver gain, fiber Raman cross section, and wavelength-dependent and local losses (at the unit input/output or near fusion splices). Using the Raman backscattered intensity traces, the distributed temperature profile has been obtained along

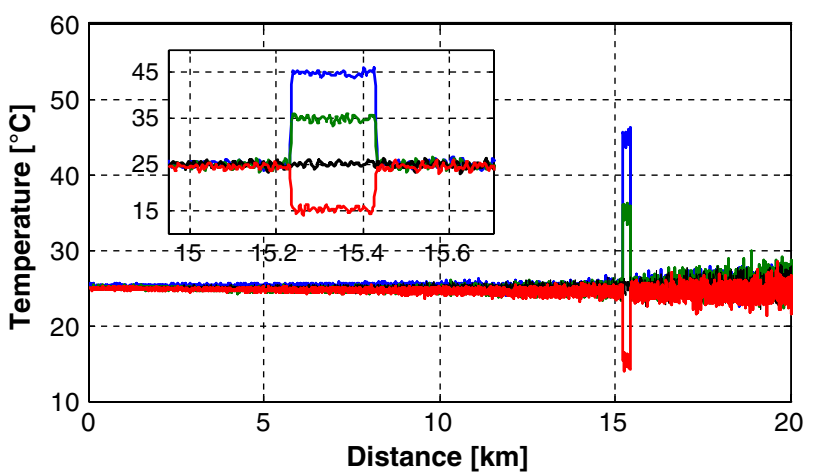

Fig. 8. (Color online) Temperature profile along $20 \mathrm{~km}$ fiber at different TCC values (red, $15^{\circ} \mathrm{C}$; black, $25^{\circ} \mathrm{C}$; green, $35^{\circ} \mathrm{C}$; blue, $\left.45^{\circ} \mathrm{C}\right)$. 

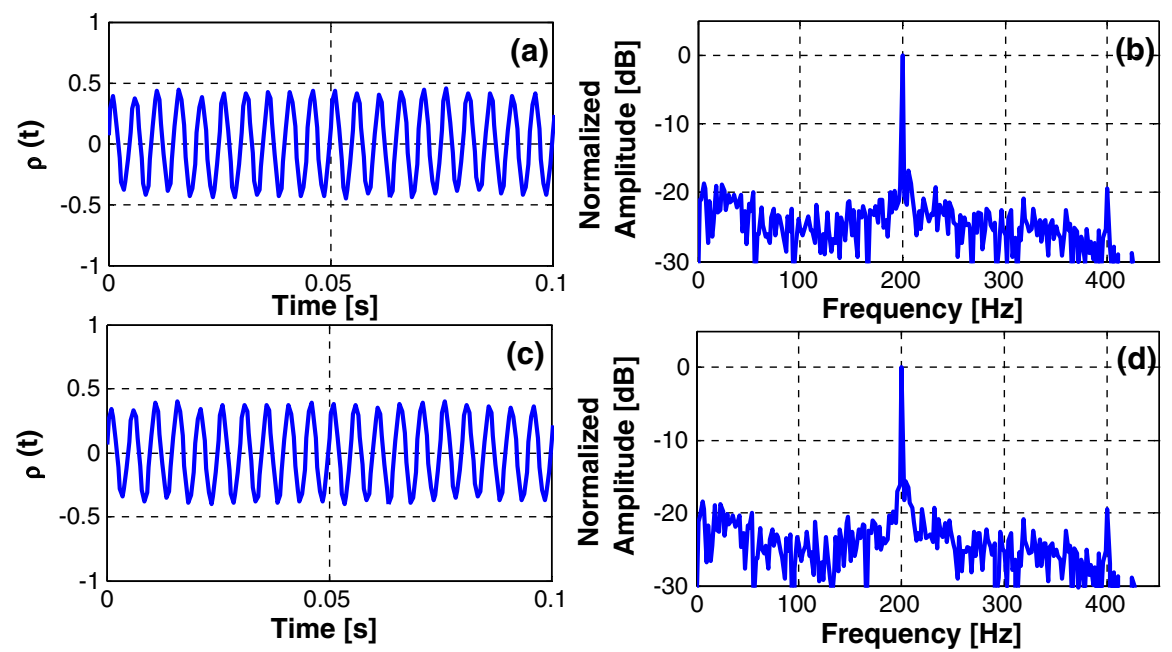

Fig. 9. (Color online) (a) Normalized time-domain trace and (b) fast Fourier transform (FFT) spectrum of S-FBG $(0.2 \mathrm{kHz}$ modulation) at $25^{\circ} \mathrm{C}$; (c) time-domain trace and (d) FFT of S-FBG at $45^{\circ} \mathrm{C}$.

$20 \mathrm{~km}$ MMF. Temperature traces at different TCC temperatures are reported in Fig. 8, clearly showing the temperature variations and the increased noise near the far fiber end. The spatial resolution (10\%-90\% temperature step response) was estimated to be $2.7 \mathrm{~m}$ at the far fiber end, mainly due to the temporal broadening of pulse and backscattered light components induced by modal dispersion throughout the $20 \mathrm{~km}$ fiber length. The temperature resolution has been estimated as the standard deviation of noise in the temperature trace, attaining $1^{\circ} \mathrm{C}$ resolution at $20 \mathrm{~km}$ distance.

Regarding simultaneous strain measurements, the acquired sinusoidal traces induced by the applied dynamic strain to S-FBG (sinusoidal waveform, $0.2 \mathrm{kHz})$ are reported in the graphs of Figs. 9(a) and 9(c); such graphs report the dynamic strain reconstruction (at $25^{\circ} \mathrm{C}$ and $45^{\circ} \mathrm{C}$ ), obtained with the interrogation function described by Eq. (4) and using the temperature information from the $\bar{R} \mathrm{DTS}$. As is evident from the figure, the final reconstructed dynamic strain trace shows very good agreement with the known strain waveform applied to the PZT. Moreover, the dynamic strain acquired with the proposed technique clearly appears to be independent on environmental temperature; actually, Fig. 9 reports about two different strain measurements [Figs. 9(a)-9(d)] obtained with the same strain conditions (same applied strain waveform at PZT) but under different temperature conditions (i.e., TCC at $25^{\circ} \mathrm{C}$ for Figs. 9(a) and 9(b), at $45^{\circ} \mathrm{C}$ for Figs. 9(c) and $9(\mathrm{~d})$. The fast Fourier transform (FFT) of the measured strain traces with FBG temperature of $25^{\circ} \mathrm{C}$ and $45^{\circ} \mathrm{C}$ is also reported in Figs. 9(b) and 9(d), respectively. Based on strain measurements, the dynamic strain resolution of our system was estimated to be about $7.8 \mathrm{n} \varepsilon /(\mathrm{Hz})^{1 / 2}$. Harmonic components are clearly visible in the amplitude spectrum, and a slight nonlinear behavior of the interrogation technique can be also observed due to the presence of (significantly smaller) spurious spectral components in addition to the fundamental one (the cross talk from harmonic components is in any case smaller than $-20 \mathrm{~dB}$ ).

\section{Conclusion}

In conclusion, we have proposed and implemented a hybrid sensor scheme, integrating SpRS and FBGbased discrete sensing employing a single interrogation unit. We have showed that the system is able to simultaneously perform distributed temperature evaluation and dynamic discrete strain measurements using an integrated reading unit with shared source and receiver stages. Experimental results show a temperature resolution better than $1^{\circ} \mathrm{C}$ with $2.7 \mathrm{~m}$ spatial resolution at a $20 \mathrm{~km}$ distance as well as a dynamic strain resolution of $7.8 \mathrm{n} \varepsilon / \sqrt{ } \mathrm{Hz}$ at $0.2 \mathrm{kHz}$ repetition rate (the Nyquist limit for a $20 \mathrm{~km}$ long fiber is $2.5 \mathrm{kHz}$ ). The proposed technique provides a high-performance solution for many applications where both dynamic and distributed measurements are simultaneously required.

\section{References}

1. K. T. V. Grattan and T. Sun, "Fiber optic sensor technology: an overview," Sens. Actuators 82, 40-61 (2000).

2. B. Culshaw, "Fiber-optic sensing: a historical perspective," J. Lightwave Technol. 26, 1064-1078 (2008).

3. B. Culshaw, "Fibre optic sensor technology-an engineering reality or a scientific opportunity?" Proc. SPIE 7653, 765304 (2010), invited paper.

4. F. T. S. Yu and S. Yin, Fiber Optic Sensors (Dekker, 2002), Chaps. 4 and 5 .

5. B. Gholamzadeh and H. Nabovati, "Fiber optic sensors," World Acad. Sci. Eng. Technol. 42, 297-307 (2008).

6. A. D. Kersy, M. A. Davis, H. J. Patrick, M. LeBlan, and K. P. Koo, "Fiber grating sensors," J. Lightwave Technol. 15, 1442-1463 (1997).

7. C. C. Chan, W. Jin, and H. L. Ho, "Performance analysis of a time-division-multiplexed fiber Bragg grating sensor array by use of a tunable laser source," IEEE J. Sel. Top. Quantum Electron. 6, 741-749 (2000).

8. Y. Wang, J. Gong, D. Y. Wang, B. Dong, and W. Bi, "A quasidistributed sensing network with time-division-multiplexed fiber Bragg gratings," IEEE Photon. Technol. Lett. 23, 70-72 (2011). 
9. N. Anscombe and O. Graydon, eds., "Optical Fibre Sensors," Nat. Photon. Technology Focus 2, 143-158 (2008).

10. M. A. Soto, P. K. Sahu, S. Faralli, G. Bolognini, and F. Di Pasquale, "Distributed temperature sensor system based on Raman scattering using correlation-codes," Electron. Lett. 43, 862-864 (2007).

11. A. Signorini, S. Faralli, M. A. Soto, G. Sacchi, F. Baronti, R. Barsacchi, A. Lazzeri, R. Roncella, G. Bolognini, and F. Di Pasquale, " 40 km long-range Raman based distributed temperature sensor with meter-scale spatial resolution," in Optical Fiber Communication Conference, OSA Technical Digest (CD) (Optical Society of America, 2010), paper OWL2.

12. K. O. Hill and G. Meltz, "Fiber Bragg grating technology fundamentals and overview," J. Lightwave Technol. 15, 1263-1276 (1997).

13. A. Othonos and K. Kalli, Fiber Bragg Gratings, Fundamentals and Applications in Telecommunications and Sensing (Artech House, 1999), Chap. 3. 